\title{
Increased expression of mitochondrial transcription factor $A$ and nuclear respiratory factor-1 predicts a poor clinical outcome of breast cancer
}

\author{
WEI GAO ${ }^{1,2^{*}}$, MEIHONG WU ${ }^{2 *}$, NING WANG ${ }^{2}$, YINGYI ZHANG ${ }^{2}$, \\ JING HUA $^{2}$, GUSHENG TANG ${ }^{3}$ and YAJIE WANG ${ }^{2}$ \\ ${ }^{1}$ Department of Radiation Oncology, Shanghai 9th People's Hospital Affiliated to \\ Shanghai Jiao Tong University School of Medicine; Departments of ${ }^{2}$ Oncology and \\ ${ }^{3}$ Hematology, Changhai Hospital, The Second Military Medical University, Shanghai 200433, P.R. China
}

Received July 6, 2017; Accepted August 11, 2017

DOI: $10.3892 / \mathrm{ol} .2017 .7487$

\begin{abstract}
Nuclear respiratory factor-1 (Nrf1) and mitochondrial transcription factor A (TFAM) are involved in the regulation of a variety of mitochondrial functional genes, which are associated with decreased sensitivity of tumor cells to chemotherapy. However, the expression status of Nrf1 and TFAM, as well as their clinical significance in breast cancer, is unknown. In the present study, tumor tissues and corresponding adjacent normal tissues were collected from 336 patients with breast cancer, and Nrf1 and TFAM expression was analyzed by immunohistochemistry using a tissue microarray. Expression of Nrf1 and TFAM was significantly increased in breast cancer tissue compared with adjacent normal tissues. In addition, patients positive for Nrf1 or TFAM had a poorer clinical prognosis than patients who were negative, and those positive for Nrf1 and TFAM had the shortest survival time. These results suggest that Nrf1 and TFAM are potential biomarkers for the determination of individualized therapy and the prognosis of breast cancer, and molecular
\end{abstract}

Correspondence to: Professor Gusheng Tang, Department of Hematology, Changhai Hospital, The Second Military Medical University, 168 Changhai Road, Shanghai 200433, P.R. China E-mail: drake015@163.com

Professor Yajie Wang, Department of Oncology, Changhai Hospital, The Second Military Medical University, 168 Changhai Road, Shanghai 200433, P.R. China

E-mail: yajiewa0820@163.com

*Contributed equally

Abbreviations: DDP, cisplatin; TFAM, mitochondrial transcription factor A; Nrf1, nuclear respiratory factor-1; TMA, tissue microarray; IHC, immunohistochemistry; OS, overall survival; ATP, adenosine triphosphate

Key words: nuclear respiratory factor-1, mitochondrial transcription factor A, breast cancer, prognosis targeting of Nrf1 and TFAM is a promising strategy for the sensitization of breast cancer cells to chemotherapeutics.

\section{Introduction}

Breast cancer is one of the most common malignancies globally and a major cause of cancer-associated mortality in women (1). Cisplatin (DDP) is the first line chemotherapeutic drug for solid tumor types, including breast cancer. However, DDP resistance is one of the current challenges facing the management of breast cancer patients. DDP primarily acts to form complexes with mitochondrial DNA (mtDNA), leading to mitochondria damage and concomitant cell death through the intrinsic apoptosis pathway $(2,3)$. Furthermore, DDP reduces mtDNA copies and increases the sensitivity of tumor cells to chemotherapy (4). Thus, dysregulation of mtDNA copy number and gene expression may be associated with the progression and prognosis of breast cancer following DDP treatment.

Mitochondrial transcription factor A (TFAM) drives the transcription and replication of mtDNA and is involved in the regulation of mtDNA copies and the maintenance and repair of mitochondrial genes (5). Therefore, TFAM is important in terms of the functional integrity of the mitochondrial respiratory chain and the maintenance of balance between anti-oxidation and oxidation (5). Nuclear respiratory factor-1 (Nrf1) may bind to the promoter of the TFAM gene to regulate TFAM expression, and may be involved the biogenesis and adenosine triphosphate (ATP) production in mitochondria $(6,7) . N R F 1$ and TFAM expression has been demonstrated to be associated with the clinical features of certain tumor types, including esophageal squamous cell carcinoma, colorectal, liver and bladder cancer (8-11). NRF1 and TFAM mRNA and protein expression have been demonstrated to be positive in patients with breast cancer compared with adjacent normal patients or in MCF-7, MDA-MB-231 and MDA-MB-453 cell lines compared with a control Hs578T cell line (12,13), but NRF1 and TFAM expression patterns in breast cancer and adjacent normal tissues, as well as their clinical significance, remain unclear. In the present study, breast cancer tissues and adjacent normal tissues were collected from 
patients, and immunohistochemistry array analysis of $\mathrm{Nrf1}$ and TFAM protein expression was performed. The results of the present study demonstrated that Nrf1 and TFAM protein expression was increased in the cancer cells of patients with different types of breast cancer, and patients who were positive for Nrf1 and TFAM had a decreased long-term survival rate compared with patients who were negative.

\section{Materials and methods}

Patients. All patients with primary breast cancer who had undergone initial surgery at the First Affiliated Hospital of The Second Military Medical University (Changhai Hospital, Shanghai, China) between January 2009 and June 2010 were screened for enrolment in the present study by reviewing electronic charts. Patients who presented with other primary tumor sites or who received preoperative radiotherapy or chemotherapy were excluded. A total of 388 patients were enrolled in the present study and 336 patients with complete clinical information were included for further analysis. The following variables were recorded: Patient age at diagnosis, menopausal status, largest tumor diameter, number of lymph node metastases, tumor-node-metastasis stage (TNM, NCCN Guidelines, Breast Cancer Version 3.2014) (14) and histologic grade. Clinicopathological characteristics for these patients are detailed in Table I. All tissue specimens used in the present study were obtained with written informed consent from the patients, and the Ethics Committee of Changhai Hospital granted approval for this measure and the research protocol.

Tissue microarray (TMA) and immunohistochemistry (IHC). Large core TMAs were used in order to cover a larger number of tumor cells and represent the typical pathological changes, as described previously (15). Tissues were embedded in paraffin and sectioned ( $4 \mu \mathrm{m}$ thickness) for hematoxylin (4 min) and eosin $(3 \mathrm{~min})$ staining $\left(25^{\circ} \mathrm{C}\right)$. Pathological examination was performed by an experienced pathologist, and the region of interest was marked on the basis of the HE staining results. Away from areas of ulceration and necrosis, $1.5-\mathrm{mm}$ diameter cylinders were punched from the center of the excised tumor and re-embedded into a recipient paraffin block, using a tissue-arraying instrument (Beecher Instruments, Inc., Sun Prairie, WI, USA). The microarray sections were randomly inspected, processed for HE staining and observed under a light microscope for quality control. The TMA blocks were then cut into 4-mm sections and processed for IHC.

Tissues were fixed in $4 \%$ neutral formalin at $4^{\circ} \mathrm{C}$ for $24 \mathrm{~h}$ and embedded in paraffin. Tissue sections were dewaxed and washed with $3 \%$ hydrogen peroxide at room temperature for $15 \mathrm{~min}$, followed by further washing with double distilled water for $6 \mathrm{~min}$ and PBS for $6 \mathrm{~min}$. The microarray sections were put into the microwave oven and heated until boiling in $0.01 \mathrm{~mol} / 1$ sodium citrate buffer solution ( $\mathrm{pH}$ 6.0) for $5 \mathrm{~min}$, repeated 2 times, then cooled at room temperature for $20 \mathrm{~min}$, before being washed in PBS. Sections were incubated in horse serum (10\%) at room temperature for $40 \mathrm{~min}$. Sections were then incubated in PBS containing $1 \%$ bovine serum albumin $(\mathrm{pH} 7.4)$ overnight followed by secondary antibody. The TFAM (K-18' cat. no. sc19050; goat anti-human) and Nrf1 (h-300; cat no. 33771; rabbit anti-human) antibodies (Santa Cruz Biotechnology,
Table I. Clinical characteristics of patients enrolled in the present study.

\begin{tabular}{lc}
\hline Clinicopathological variable & Value \\
\hline Median age, years (range) & $53(30-81)$ \\
Menopausal status (\%) & $152(45.2)$ \\
Premenopausal & $184(54.8)$ \\
Postmenopausal & \\
TNM stage (\%) & $104(31.0)$ \\
I & $195(58.0)$ \\
II & $37(11.0)$ \\
III & \\
Pathological diagnosis (\%) & $312(92.9)$ \\
Invasive ductal carcinoma & $24(7.1)$ \\
Non-invasive ductal carcinoma & \\
Histological grade (\%) & $16(4.8)$ \\
1 & $216(64.3)$ \\
2 & $104(30.9)$ \\
3 & \\
Erb-b2 receptor tyrosine kinase 2 status (\%) & \\
Negative & $280(83.3)$ \\
Positive & $56(16.7)$ \\
Estrogen receptor status (\%) & \\
Negative & $152(45.2)$ \\
Positive & $184(54.8)$ \\
Progesterone receptor status (\%) & \\
Negative & $198(58.9)$ \\
Positive & $138(41.1)$ \\
\hline
\end{tabular}

Inc., Dallas, TX, USA) were used at final dilutions of 1:100 and 1:1,000 overnight at $4^{\circ} \mathrm{C}$ respectively. Immunostaining was conducted using the Dako EnVision System with diaminobenzidine according to the manufacturer's protocol (Dako, Glostrup, Denmark). AperioImageScope v11.2.2.752 software (http://aperio-imagescope.software. Informer.com) was used to capture images, which were then independently evaluated by two experienced pathologists who were blind to the clinicopathological factors of the patients in the present study. Any discrepancy was resolved by consulting a third pathologist.

Semi-quantitative criteria. A semi-quantitative evaluation of Nrf1 and TFAM positive staining in IHC was performed using a previously described method (15). Briefly, five representative images from IHC for Nrf1 and TFAM were captured from each section at a high magnification (x200). The staining intensity compared with the background and the percentage of positive cells were determined in a blind manner. The percentage of positive cells was divided into five grades (percentage scores): $0,<10 ; 1,10-25 ; 2,26-50 ; 3,51-75 ;$ and $4,>75 \%$. The intensity of staining was divided into four grades (intensity scores): 0 , no staining; 1, light brown; 2, brown; and 3, dark brown. The total scores (percentage score $\mathrm{x}$ intensity score) ranged from 0 to 12 , and were divided into low expression $(0-2)$ or high expression (3-12) groups. 


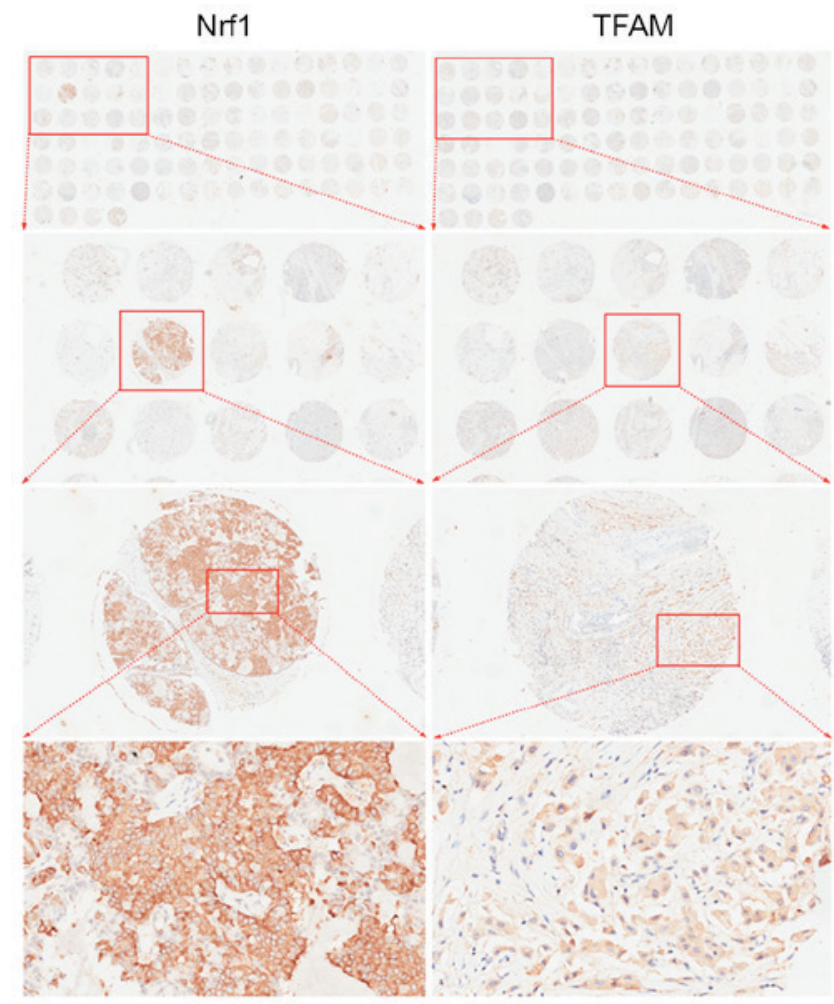

Figure 1. Preparation of tissue microarrays and images from immunohistochemistry. Tissue microarray was prepared as described in Materials and Methods. Antibodies against TFAM and Nrf1 (1:100 or 1:1,000) were employed for immunohistochemistry. AperioImageScope was used to capture images. The microarrays of each patient for Nrf1 and TFAM were screened and analyzed at different magnifications.

Clinicopathological parameters and their classifications. Estrogen receptor (ER) and progesterone receptor (PR) status were classified thus: Cells positive for ER and PR had immunoreactivity in the nuclei with or without cytoplasmic staining in IHC. The nucleus was a major site where immunoreactivity existed for ER and PR, and cells with only cytoplasmic immunoreactivity were regarded as negative for ER or PR. ER and PR expression was determined according to the percentage of positive cells: Negative, $\leq 10 \%$; positive, $>10 \%$.

Erb-b2 receptor tyrosine kinase 2 (HER2) protein expression was assessed by IHC. Cells were regarded as positive for HER2 when $3+$ was noted in IHC or $2+$ was present in IHC and simultaneous positive staining was observed in fluorescence in situ hybridization (FISH) (16). FISH analysis was performed using the PathVysion HER-2 probe kit (Abbott Pharmaceutical Co. Ltd., Lake Bluff, IL, USA). There were two fluorescent-labelled probes: LSI (locus-specific identifier) HER-2 specific for the HER-2 gene locus (17q11) and CEP (chromosome enumeration probe) 17 specific for the $\alpha$ satellite DNA sequence at the centromeric region of chromosome 17. Paraffin sections of 3-4 mm thickness using a microtome were cut and were floated in a protein-free water bath at $40^{\circ} \mathrm{C}$. The sections were mounted on poly-L-Lysine coated slides and allowed to dry. The slides were kept overnight at $56^{\circ} \mathrm{C}$. The slides were deparaffinized in xylene at room temperature for $20 \mathrm{~min}$ and dehydrated in $100 \%$ ethanol for $15 \mathrm{~min}$ at room temperature and air dried. The slides were treated with pretreatment solution (sodium thiocyanite) and protease solution for $15 \mathrm{~min}$, and were dehydrated with 70,80 and 100\% alcohol for 5 min each and air dried. The probe was denatured at $80^{\circ} \mathrm{C}$ for $5 \mathrm{~min}$, applied to the cover slip and placed in humidified chamber for overnight incubation. Post-hybridization washes were given with $0.4 \%$ sodium saline citrate 40 at $37^{\circ} \mathrm{C}$. Following removal of the cover slips the slides were dipped in post-hybridization buffer for $18 \mathrm{sec}$, dried completely in darkness and $10 \mu \mathrm{l}$ DAPI was applied. The slides were screened under a fluorescent microscope (Olympus Corporation, Tokyo, Japan) using appropriate filters (DAPI, FITC, TRITC dual and triple band pass filters). Signals were counted in at least 200 cells for both the HER-2/neu gene and chromosome 17 centromere signals under oil immersion at x1,000 magnification using recommended filters. Results are expressed as the ratio of HER-2/neu signal (orange) to centromere 17 signal (green) and the readings were read as follows; the expected ratio 1-1.8 indicates no gene amplification (negative), a ratio of $>2.2$ as HER-2/neu gene amplification (positive), and a ratio between 1.8 and 2.2 as equivocal cases. The polysomy 17 was also recorded in the cells as four spec green signals as moderate polysomy and $>4$ spec green signals as high polysomy.

Menopause was defined when one of following conditions was present: i) patients received bilateral oophorectomy; ii) patients were $\geq 60$ years old; iii) patients were $<60$ years old, but follicle-stimulating hormone (FSH) and estradiol levels were in the postmenopausal range. The menopause could not be determined in patients who were receiving luteinizing hormone-releasing hormone agonists or antagonists, and amenorrhea was not enough to determine menopause in premenopausal women receiving adjunctive chemotherapy. Under these conditions, repeated measurements of FSH and/or estradiol were required to determine whether there was a menopausal status in these patients.

Histological grading was based on the semiquantitative evaluation of three morphologic features (percentage of tubule formation, degree of nuclear pleomorphism, and accurate mitotic count in a defined field area) with a 3-point grading system (1, good, to 3 , poor). The numerical score assigned to each feature was used in the compilation of an overall grade: Scores 3-5, grade 1; scores 6-7, grade 2; scores 8-9, grade 3 (17).

The number of involved lymph nodes was scored thus: 1 involved lymph node, 1; 1-3 lymph nodes, 2; 4-9 lymph nodes, $3 ; \geq 10$ lymph nodes, 4 .

Statistical analysis. SPSS 19.0 software (IBM Corp., Armonk, NY, USA) and GraphPad software (version 5.01; GraphPad Software, Inc., La Jolla, CA, USA) were used for statistical analysis and image processing, respectively. The protein expression of Nrf1 and TFAM in breast cancer tissues were compared with adjacent normal tissues using the Wilcoxon rank sum test. Associations between Nrf1 and TFAM expression and clinicopathological features were assessed using Pearson's $\chi^{2}$ test. Multivariate analysis was performed using logistic regression. Overall survival (OS) was measured from the date of diagnosis until mortality or the last follow-up. Patients alive were censored at the time of last contact. OS rates were estimated using the Kaplan-Meier method and compared using the log-rank test. The Cox proportional 
A

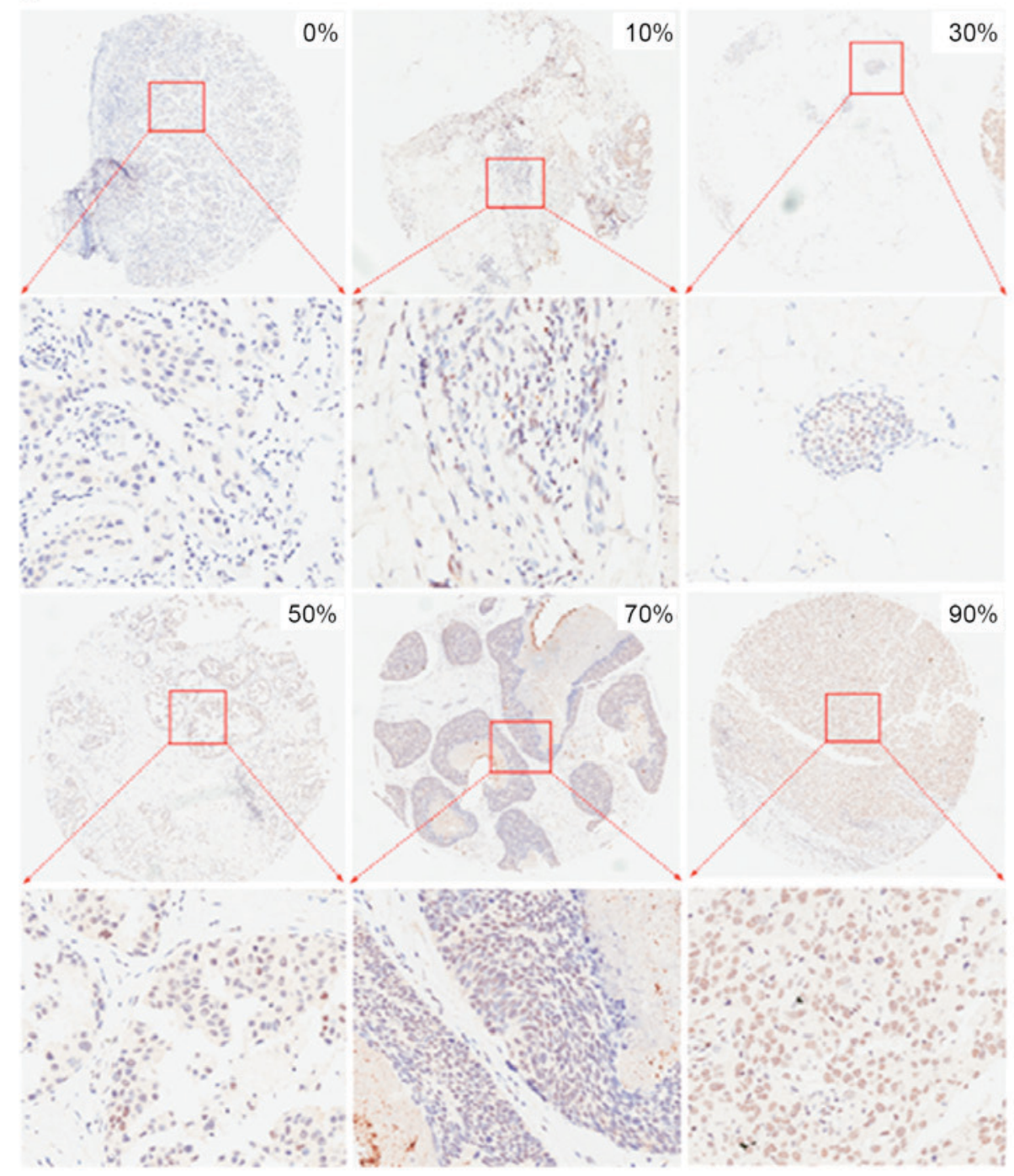

B
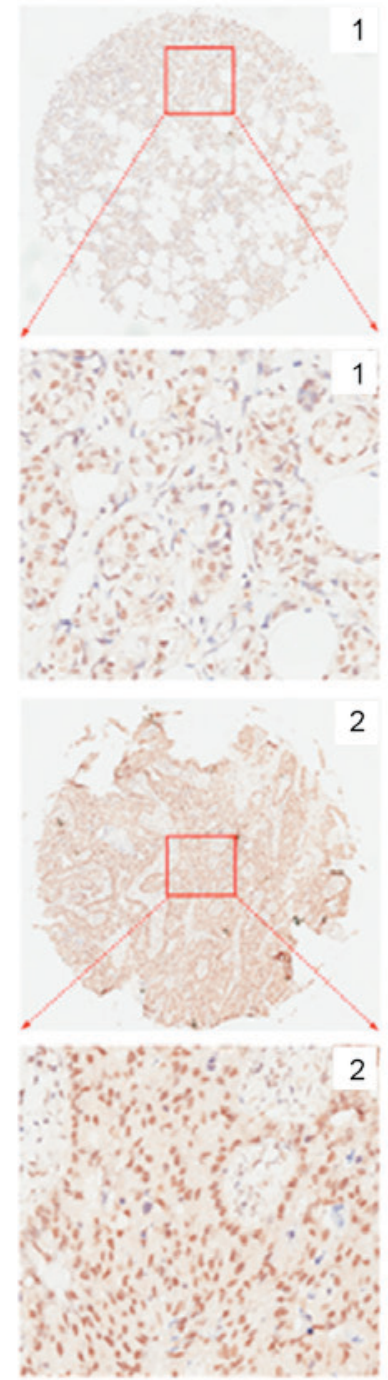

Figure 2. Images of immunohistochemistry for Nrf1 and determination of staining intensity. (A) Examples of sections with different proportions of Nrf1 positive cells; (B) Examples of images with staining intensity score of 1 and 2. In Figure A, sections without positive cells had the corresponding staining intensity of 0 . Sections with staining intensity of higher than 3 were not found in the breast cancer and adjacent normal tissues. The lower panels were the higher magnification visual field of the upper marked area.

hazards regression model was performed for univariate and multivariate survival analysis. $\mathrm{P}<0.05$ was considered to indicate a statistically significant difference.

\section{Results}

Nrf1 and TFAM expression in breast cancer and adjacent normal tissues. Nrf1 and TFAM protein expression was measured in 336 patients with TMAs. Fig. 1 presents the arrangement of representative TMAs and images captured at different magnifications following IHC analysis. Representative images with different percentages of positive cells and different staining intensities captured following immunohistochemistry for Nrf1 and TFAM are presented in Figs. 2 and 3, respectively. For sections without positive cells $(0 \%)$, the staining intensity was also classified as 0 . The majority of Nrf1-positive cells had brown staining in the nuclei (Fig. 2) with only a fraction of cancer cells expressing Nrf1 in the cytoplasm (Fig. 1) or in the nuclei and cytoplasm (data not shown). TFAM expression was observed in the cytoplasm of breast cancer cells (Fig. 3).

The association between Nrf1 and TFAM expression was evaluated in 336 patients. Paired $\chi^{2}$ tests revealed that Nrf1 and TFAM expression were not significantly associated $(\kappa=0.057, \mathrm{P}=0.258)$. The positive rate of $\mathrm{Nrf1}$ expression was significantly increased compared with TFAM in breast cancer cells $(75.3 \%$ compared with $57.1 \%$; $\mathrm{P}<0.001)$. Nrf1 binds to the promoter of TFAM to initiate TFAM expression $(6,7)$. The results of the present study demonstrated that the alteration of Nrf1 expression was not parallel to that of TFAM in breast cancer cells, suggesting that, besides Nrf1, there are additional mechanisms regulating TFAM expression.

Nrf1 and TFAM expression is increased in breast cancer tissues compared with adjacent normal tissues. To investigate the involvement of Nrf1 and TFAM in the occurrence and development of breast cancer, Nrf1 and TFAM expression was compared in breast cancer tissues and in the corresponding 
A
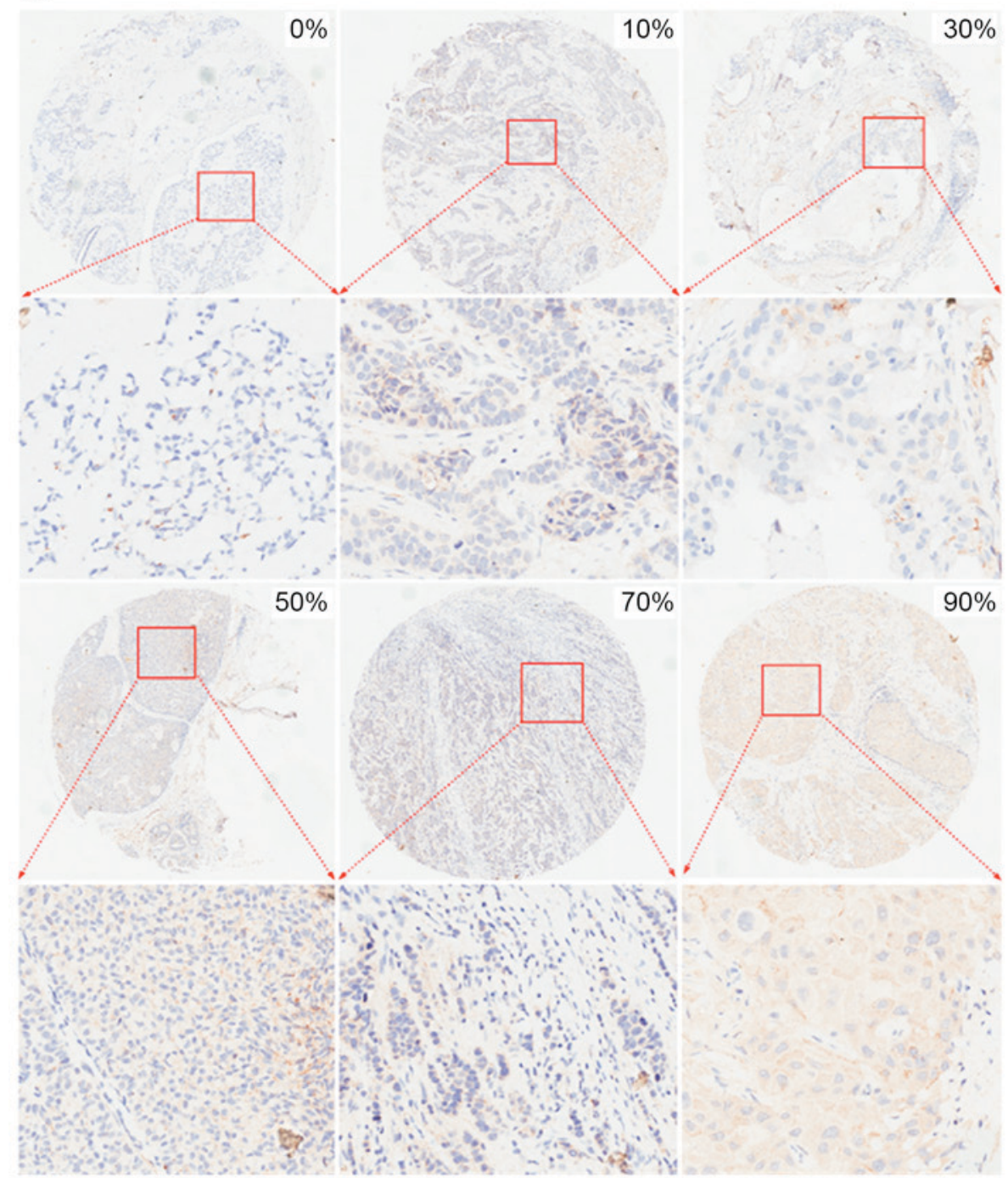

B

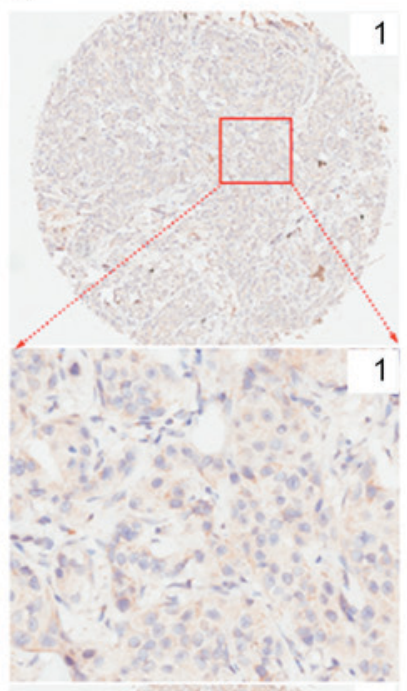

2

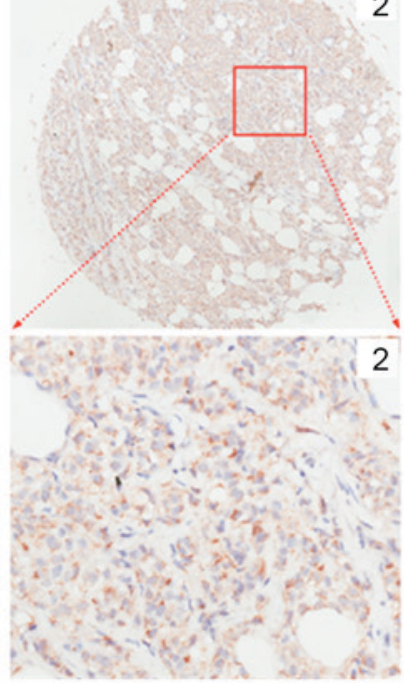

Figure 3. Images of immunohistochemistry for TFAM and determination of staining intensity. (A) sections with different proportions of TFAM positive cells; (B) images with staining intensity score of 1 and 2 . In Figure A, sections without positive cells had the corresponding staining intensity of 0 . Sections with staining intensity of higher than 3 were not found in the breast cancer and adjacent normal tissues. The lower panels were the higher magnification visual field of the upper marked area.

adjacent normal tissues from 41 patients (Table II). For Nrf1 positive cells, the mean and median integrations were 1.878 [95\% confidence interval (CI): 1.368-2.388] and 2.0 (25-75th percentile range: $0-3.5)$ in breast cancer tissue, and 0.756 (95\% CI: 0.405-1.107) and 0 (25-75th percentile range: 0-2) in the adjacent normal tissues (Fig. 4A). Wilcoxon rank sum tests revealed significant differences in the mean and median integrations between breast cancer and adjacent normal tissues, with more Nrf1 positive cells being observed in the breast cancer tissue compared with normal tissues ( $\mathrm{P}<0.0001$; Fig. 4B). For TFAM-positive cells, the mean and median integrations were 2.488 (95\% CI: 1.686-3.290) and 3.0 (25-75th percentile range: $0-4)$ in the breast cancer tissue, and 1.366 (95\% CI: 0.899-1.833) and 2.0 (25-75th percentile range: $0-2)$ in the adjacent normal tissues (Fig. 4C). Wilcoxon rank sum tests revealed that the number of TFAM-positive cells in breast cancer tissue was significantly higher than that in the adjacent normal tissue $(\mathrm{P}=0.0126$; Fig. 4D). These results demonstrated that Nrf1 and TFAM protein expression were significantly increased in breast cancer tissue compared with adjacent normal tissues, suggesting that Nrf1 and TFAM may be involved in the tumorigenesis of breast cancer and are potential targets for the therapy of breast cancer.

Association of Nrfl and TFAM expression with clinicopathological features of patients with breast cancer. Next, the associations between Nrf1 and TFAM protein expression and the clinicopathological features of patients with breast cancer were analyzed (Table III). The results revealed that Nrf1 expression was significantly associated with age, tumor size, number of metastatic lymph nodes, tumor stage, tumor grade and HER2, ER and PR expression status, but there was no significant association with menopause status or pathological type of breast cancer. Patients $<45$ years old had significantly increased Nrf1 expression compared with older patients (84.7 vs. $72.7 \%)$. Nrf1 expression in patients with a tumor size $>2 \mathrm{~cm}$, more metastatic lymph nodes or breast cancer at $>$ TNM stage II or $>$ grade 2 was significantly increased. 
Table II. Clinical characteristics of the 41 patients where expression of Nrf1 and TFAM was evaluated in cancer tissues and adjacent normal tissues.

\begin{tabular}{|c|c|}
\hline Clinicopathological variable & Value \\
\hline Median age, years (range) & $54(41-76)$ \\
\hline \multicolumn{2}{|l|}{ Menopausal status (\%) } \\
\hline Premenopausal & $13(31.7)$ \\
\hline Postmenopausal & $28(68.3)$ \\
\hline \multicolumn{2}{|l|}{ TNM stage $(\%)$} \\
\hline I & $19(46.3)$ \\
\hline II & $18(13.9)$ \\
\hline III & $4(9.8)$ \\
\hline \multicolumn{2}{|l|}{ Pathological diagnosis (\%) } \\
\hline Invasive ductal carcinoma & $35(85.4)$ \\
\hline Non-invasive ductal carcinoma & $6(14.6)$ \\
\hline \multicolumn{2}{|l|}{ Histological grade $(\%)$} \\
\hline 1 & $0(0.0)$ \\
\hline 2 & $28(68.3)$ \\
\hline 3 & $13(31.7)$ \\
\hline \multicolumn{2}{|c|}{ Erb-b2 receptor tyrosine kinase 2 status (\%) } \\
\hline Negative & $33(80.5)$ \\
\hline Positive & $8(19.5)$ \\
\hline \multicolumn{2}{|l|}{ Estrogen receptor status (\%) } \\
\hline Negative & $36(87.8)$ \\
\hline Positive & $5(12.2)$ \\
\hline \multicolumn{2}{|l|}{ Progesterone receptor status $(\%)$} \\
\hline Negative & $37(90.2)$ \\
\hline Positive & $4(9.8)$ \\
\hline
\end{tabular}

Patients positive for HER2, ER and PR had markedly higher Nrf1 expression than patients negative for these factors.

TFAM expression was also associated with the tumor size and HER2, ER and PR status. Patients positive for HER2, ER and PR had increased TFAM expression compared with those who were not, while patients with tumor size of $>2 \mathrm{~cm}$ had a slight reduction in TFAM expression. These results indicated that Nrf1 and TFAM expression is associated with factors associated with the clinical outcome of patients with breast cancer, and thus may serve as important predictors for patients for breast cancer.

Association between Nrfl and TFAM expression and the clinical prognosis of breast cancer. To further evaluate the associations between Nrf1 and TFAM expression and the clinical prognosis of patients with breast cancer, the relationship between Nrf1 and TFAM expression and survival time was assessed. Survival analysis revealed that patients with breast cancer with higher Nrf1 or TFAM expression had a poorer clinical prognosis, as demonstrated by a shorter survival time (Fig. 5A and B). At the predesigned time points, the expected survival time was 91.76 months (95\% CI: 86.56-96.96, $\mathrm{P}=0.008$ ) in patients positive for $\mathrm{Nrf1}$, which was significantly shorter than in Nrf1-negative patients
(117.55 months; 95\% CI: 109.95-125.15). The expected survival time was 85.56 months (95\% CI: 79.78-91.35) in patients positive for TFAM, which was significantly shorter than in TFAM-negative patients (113.46 months; 95\% CI: 106.88-120.035, $\mathrm{P}=0.001)$. Furthermore, patients positive for Nrf1 and TRAM had the shortest survival time (82.07 months, 95\% CI: 75.154-88.99) and those negative for both Nrf1 and TRAM had the longest survival time (121.82 months, 95\% CI: 113.01-130.63; $\mathrm{P}<0.001$; Fig. 5C). These results revealed that Nrf1 or TFAM-positive patients had a shorter survival time, and Nrf1 and TRAM-double positive patients had a poorer clinical outcome. To explore whether Nrf1 and TFAM expression was an independent predictor of survival, univariate and multivariate analyses were performed using the Cox proportional hazards model. As presented in Table IV, Nrf1 expression was independently associated with the prognosis of patients $(\mathrm{P}=0.034)$, together with TFAM $(\mathrm{P}=0.048)$, HER2 $(\mathrm{P}=0.042), \mathrm{PR}(\mathrm{P}=0.039)$ and TNM stages $(\mathrm{P}=0.028)$. These results suggest that Nrf1 and TFAM may serve as predictors for breast cancer prognosis, and the combined use of Nrf1 and TFAM may assist risk stratification and subsequent therapy of breast cancer.

\section{Discussion}

The mitochondrion is an important organelle in eukaryotic cells, and is the site where intracellular oxidative phosphorylation and ATP synthesis occur. Each mitochondrion is estimated to contain 2-10 copies of mtDNA, which are unique extranuclear genetic materials that self-replicate and are involved in transcription and encoding. The synthesis and degradation of mtDNA is very rapid, independent of the cell cycle, and is controlled by complex regulatory mechanisms. Furthermore, mtDNA has poor stability, its synthesis and degradation is susceptible to exogenous factors, and the mtDNA mutation rate is 10 times greater than that of nuclear DNA. Mitochondria are also involved in important cellular activities including cell differentiation, communication, apoptosis and energy metabolism, but the specific mechanisms underlying these are still poorly understood (4). An analysis of genome-wide transcriptional profiling data revealed that $\sim 40$ transcripts were significantly elevated in human breast cancer cells compared with adjacent stromal tissues, and immunohistochemistry revealed that 15 markers of mitochondrial biogenesis and/or mitochondrial translation (including Nrf1 and TFAM) were highly expressed in epithelial breast cancer cells (18). In estrogen-induced breast carcinogenesis, Nrf1 expression markedly increased in MCF-10A cells (19). Furthermore, the mRNA and protein levels of Nrf1 and/or TFAM were revealed to dramatically increase in different types of breast cancer $(12,13)$. These results indicate that Nrf1 and TFAM expression may be associated with the occurrence and development of breast cancer.

TFAM is encoded by a gene mapped to chromosome10q21 and belongs to the high mobility group box family. Human TFAM may directly bind to the heavy and light chain promoters to activate the transcription of mitochondrial genes and regulate mtDNA copies, which is essential for the functional integrity of mitochondrial respiratory chain (20-22). 
Table III. Associations between Nrf1 and TFAM expression and clinicopathological features.

\begin{tabular}{|c|c|c|c|c|c|c|}
\hline \multirow[b]{2}{*}{ Clinicopathological variable } & \multicolumn{3}{|c|}{ Nrf1 } & \multicolumn{3}{|c|}{ TFAM } \\
\hline & Negative & Positive & $\chi^{2} / \mathrm{P}$-value & Negative & Positive & $\chi^{2} / \mathrm{P}$-value \\
\hline Age at diagnosis, years & & & $4.376 / 0.036$ & & & $0.589 / 0.443$ \\
\hline$\leq 45$ & 11 & 61 & & 28 & 44 & \\
\hline$>45$ & 72 & 192 & & 116 & 148 & \\
\hline Menopausal status & & & $2.769 / 0.096$ & & & $0.001 / 0.975$ \\
\hline Premenopausal & 31 & 121 & & 65 & 87 & \\
\hline Postmenopausal & 52 & 132 & & 79 & 105 & \\
\hline Tumor size, cm & & & $25.925 /<0.001$ & & & $28.329 /<0.001$ \\
\hline$\leq 2$ & 40 & 80 & & 54 & 66 & \\
\hline $2-5$ & 36 & 148 & & 63 & 121 & \\
\hline$\geq 5$ & 7 & 25 & & 27 & 5 & \\
\hline Lymph node metastasis & & & $10.763 / 0.005$ & & & $5.609 / 0.061$ \\
\hline $0-1$ & 67 & 198 & & 106 & 159 & \\
\hline 2 & 16 & 30 & & 27 & 19 & \\
\hline $3-4$ & 0 & 25 & & 11 & 14 & \\
\hline Tumor-node-metastasis stage & & & $35.243 /<0.001$ & & & $3.283 / 0.194$ \\
\hline I & 34 & 70 & & 43 & 61 & \\
\hline II & 13 & 182 & & 80 & 115 & \\
\hline III & 5 & 32 & & 21 & 16 & \\
\hline Pathological diagnosis & & & $2.276 / 0.131$ & & & $<0.001 / 1.000$ \\
\hline Invasive ductal carcinoma & 74 & 238 & & 135 & 180 & \\
\hline Non-invasive ductal carcinoma & 9 & 15 & & 9 & 12 & \\
\hline Histology grade & & & $2.157 / 0.034$ & & & $2.379 / 0.304$ \\
\hline 1 & 6 & 10 & & 4 & 12 & \\
\hline 2 & 49 & 167 & & 89 & 127 & \\
\hline 3 & 28 & 76 & & 47 & 57 & \\
\hline Erb-b2 receptor tyrosine kinase 2 status & & & $5.379 / 0.020$ & & & $5.600 / 0.018$ \\
\hline Negative & 76 & 204 & & 112 & 168 & \\
\hline Positive & 7 & 49 & & 32 & 24 & \\
\hline Estrogen receptor status & & & $10.015 / 0.002$ & & & $26.675 /<0.001$ \\
\hline Negative & 50 & 102 & & 64 & 88 & \\
\hline Positive & 33 & 151 & & 80 & 104 & \\
\hline Progesterone receptor status & & & $13.124 /<0.001$ & & & $35.754 /<0.001$ \\
\hline Negative & 63 & 135 & & 87 & 111 & \\
\hline Positive & 20 & 118 & & 7 & 81 & \\
\hline
\end{tabular}

TFAM overexpression results in an increase in mtDNA copies, while TFAM knockout resulted in a decrease in several murine organs and tissues $(22,23)$. Cells with TFAM deficiency demonstrated a significant increase in cell apoptosis $(21,24)$. At the initial stage of p53 dependent apoptosis, p53 may bind to TFAM to strengthen the binding of TFAM to DDP-damaged mtDNA, which may contribute to the maintenance and repair of mtDNA structure and regulate p53-mediated cell apoptosis (25). Yoshida et al (26) confirmed that TFAM preferentially recognized DDP-damaged DNA. These results suggest that TFAM may protect mtDNA against DDP-induced damage and promote repair of the damaged mtDNA, implying that a high TFAM expression may contribute to the resistance of cancer cells to DDP-based chemotherapy, thereby influencing the clinical outcome.

The proximal promoter of the TFAM gene contains the binding sites of specificity protein 1 (SP1), Nrf1 and nuclear respiratory factor-2 (Nrf2). Nrf1 binding is a determinant for the bioactivity of TFAM. Mutation or methylation of the Nrf1 binding site on the TFAM gene may significantly reduce TFAM transcription, while Nrf1 phosphorylation may stimulate it. Furthermore, SP1 and Nrf2-induced TFAM transcription also requires Nrf1 binding $(27,28)$. Thus, Nrf1 is functionally associated with TFAM and is regarded as an important regulator of TFAM. Nrf1, together with TFAM, may regulate the expression of a variety of mitochondrial functional proteins, including 
A

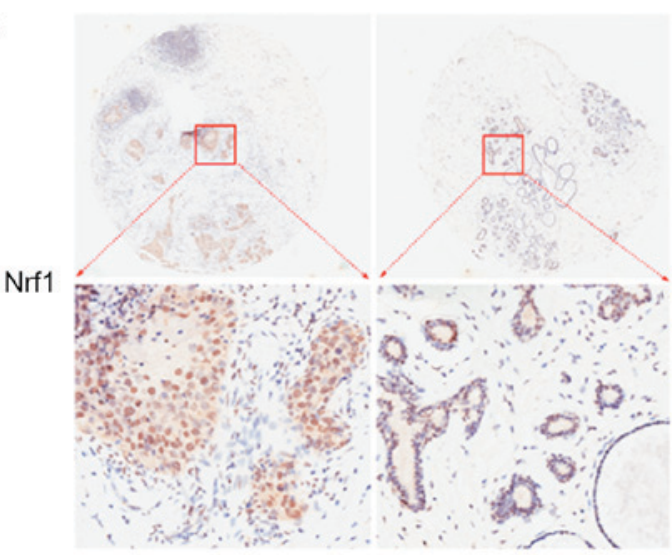

C

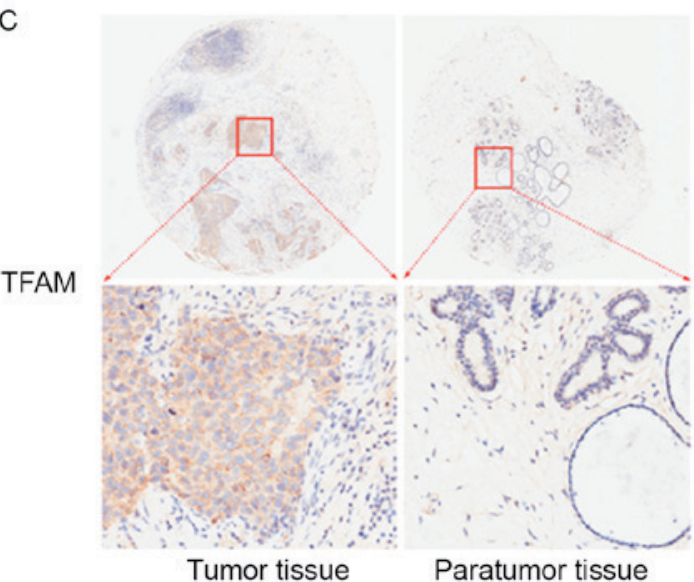

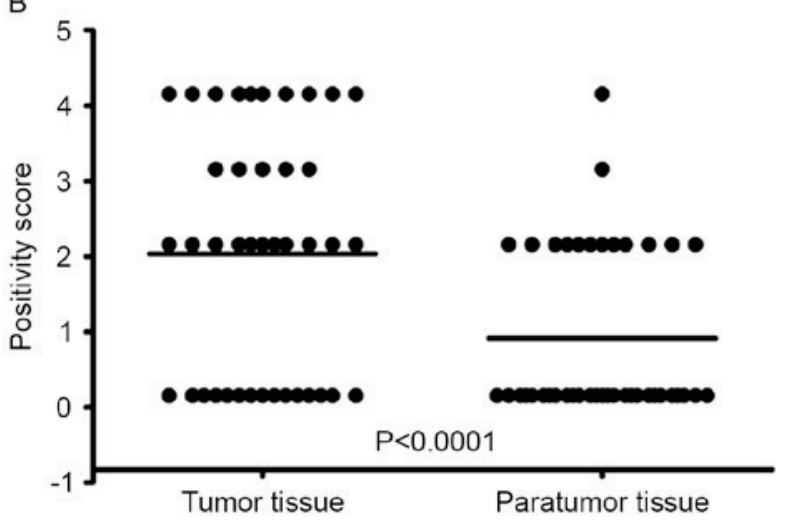

D

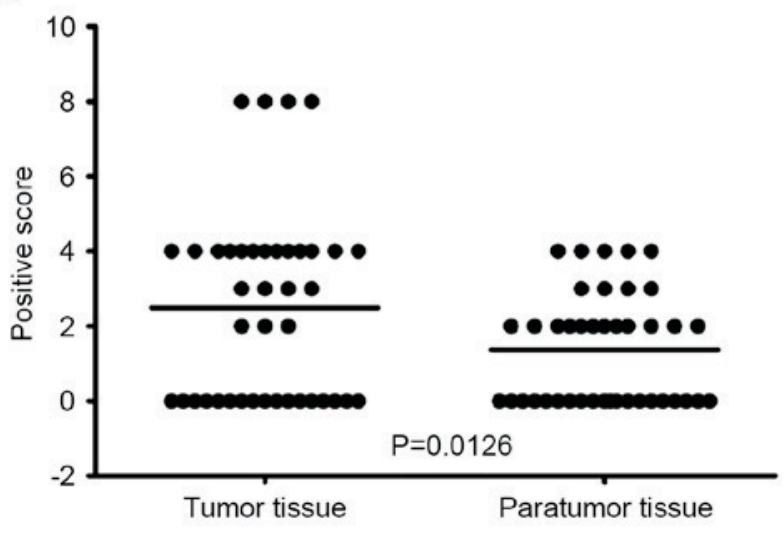

Figure 4. Nrf1 and TFAM expression in breast cancer and adjacent normal tissues. Nrf1 and TFAM expressions were measured in the breast cancer and corresponding adjacent normal tissues of 41 patients, and then scored. (A and C) Examples showing Nrf1 and TFAM expression of the same patient; (B and D) Significant higher total expression scores of Nrf1 and TFAM were identified in breast cancer than adjacent normal tissues (Wilcoxon rank sum test, Nrf1, $\mathrm{P}<0.001$; TFAM, $\mathrm{P}=0.0126)$. Horizontal line in $\mathrm{B}$ and $\mathrm{D}$ indicates the means in different groups.

A

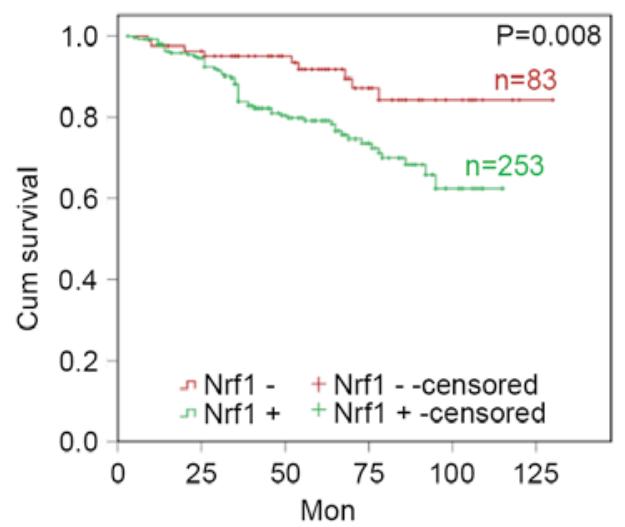

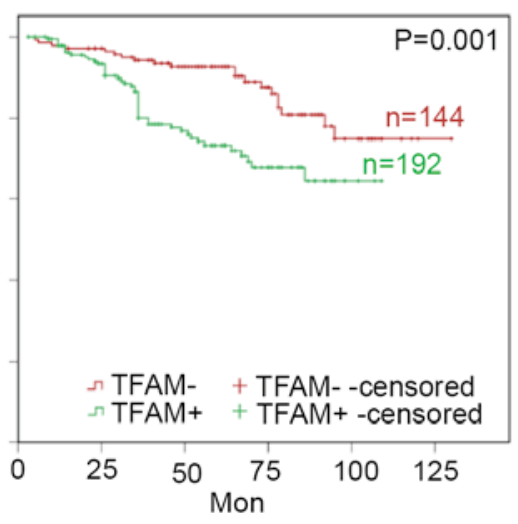

C

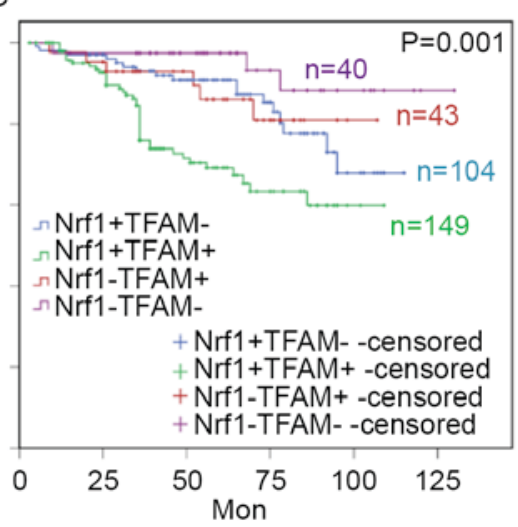

Figure 5. Patients with high Nrf1 and TFAM expressions had a poor prognosis. Univariate analysis showed breast cancer patients positive for Nrf1 (A, P=0.008) or TFAM (B, $\mathrm{P}=0.001)$ had a significantly shorter survival time as compared to patients negative for Nrf1 or TFAM. Moreover, the survival time was the shortest in patients positive for both Nrf1 and TFAM and the highest in those negative for both Nrf1 and TFAM $(C, P<0.001)$.

those involved in oxidative phosphorylation, complex I-V, mtDNA transcription and replication, protein import and assembly, ion channels, shuttle and translation (29). Thus, evaluation of Nrf1 and TFAM expression in breast cancer and adjacent normal tissues, and their predictive value, will assist in the identification of novel targets for the individualized therapy of breast cancer, the risk stratification of breast cancer, and the improvement of clinical therapeutic efficacy and prognosis of patients with breast cancer.

In the present study, Nrf1 and TFAM expression was assessed in breast cancer tissues and adjacent normal tissues of 336 patients by immunohistochemistry, and the associations with clinicopathological features were further evaluated in these patients. The results of the present study revealed that $\mathrm{Nrf1}$ 
Table IV. Univariate and multivariate analysis of overall survival the enrolled 336 patients with breast cancer.

\begin{tabular}{|c|c|c|c|c|c|c|}
\hline \multirow[b]{2}{*}{ Factor } & \multicolumn{3}{|c|}{ Univariate analysis } & \multicolumn{3}{|c|}{ Multivariate analysis } \\
\hline & HR & $95 \% \mathrm{CI}$ & P-value & HR & $95 \% \mathrm{CI}$ & P-value \\
\hline Age & 1.119 & $0.926-1.313$ & 0.854 & & & \\
\hline Menopausal & 1.959 & $0.793-3.125$ & 0.485 & & & \\
\hline Histology grade & 2.397 & $0.581-4.212$ & 0.092 & & & \\
\hline TNM & 6.365 & $3.884-15.845$ & 0.005 & 3.645 & $0.968-7.743$ & 0.028 \\
\hline HER2 & 3.245 & $1.263-6.235$ & 0.012 & 2.026 & $0.668-5.035$ & 0.042 \\
\hline ER & 2.826 & $1.035-5.014$ & 0.045 & 1.869 & $0.893-3.254$ & 0.051 \\
\hline PR & 2.956 & $1.156-5.426$ & 0.031 & 1.926 & $0.998-3.547$ & 0.039 \\
\hline Nrf1 & 4.896 & $2.552-13.312$ & 0.001 & 3.154 & $1.021-7.216$ & 0.034 \\
\hline TFAM & 3.936 & $0.031-0.435$ & 0.009 & 2.536 & $0.649-5.268$ & 0.048 \\
\hline
\end{tabular}

TNM, tumor-node-metastasis; HER2, erb-b2 receptor tyrosine kinase 2; ER, estrogen receptor; PR, progesterone receptor; Nrf1, nuclear respiratory factor-1; TFAM, mitochondrial transcription factor A.

and TFAM expression was significantly higher in breast cancer tissue compared with adjacent normal tissues, suggesting that high expression of Nrf1 and TFAM may contribute to the high energy metabolism of breast cancer cells. In estrogen-induced breast carcinogenesis in MCF-10A cells, Nrf1 expression markedly increased, further supporting the conclusion that Nrf1 and TFAM are involved in the tumorigenesis of breast cancer (18). However, further investigation revealed that the Nrf1-positive rate was higher than the TFAM-positive rate in breast cancer tissues. This may be explained by the fact that Nrf1-induced transcription of TFAM is associated with not only Nrf1 expression levels, but also Nrf1 phosphorylation and methylation $(27,28)$.

Statistical analysis revealed that high expression of Nrf1 and TFAM was associated with several clinicopathological features associated with the prognosis of breast cancer. Patients with increased Nrf1 or TFAM expression had a shorter survival time, and survival was shortest in patients positive for Nrf1 and TFAM. Considering that Nrf1 is able to regulate TFAM expression, Nrf1 may effectively activate TFAM and its downstream signaling pathway in patients positive for Nf1 and TFAM. Cancer cells in these patients may have a more active energy metabolism, increased proliferation and invasion, and elevated resistance to routine radiotherapy or chemotherapy, resulting in a poorer clinical prognosis. The significant associations with HER2, ER, PR expression status and Nf1 or TFAM expression indicates that HER2, ER and PR may be involved in the protective mechanism of TFAM against DDP-mediated mtDNA damage. More mechanistic studies, however, are required to further clarify the potential associations between expression of these proteins and their function in the etiology of breast cancers.

In summary, to the best of our knowledge, the results of the present study are the first to provide clinical evidence that Nrf1 and/or TFAM expression significantly increase in breast cancer compared with adjacent normal tissues. In addition, patients positive for Nrf1 or TFAM have a relatively poor clinical prognosis, and those positive for Nrf1 and TFAM have the shortest survival time. These results suggest that Nrf1 and/or TFAM expression may be useful as a parameter for the determination of individualized therapy and the prognosis of breast cancer, and Nrf1 and TFAM may be novel targets for the development of targeted therapies to reduce the resistance of breast cancer cells to chemotherapeutics.

\section{Acknowledgements}

The present study was supported by the Natural Science Foundation of Shanghai Science and Technology Commission (grant no. 14ZR1408800) and the National Natural Science Foundation of China (grant no. 81102249).

\section{References}

1. Siegel RL, Miller KD and Jemal A: Cancer statistics, 2015. CA Cancer J Clin 65: 5-29, 2015.

2. Renatus M, Stennicke HR, Scott FL, Liddington RC and Salvesen GS: Dimer formation drives the activation of the cell death protease caspase 9. Proc Natl Acad Sci USA 98: 14250-14255, 2001.

3. Acehan D, Jiang X, Morgan DG, Heuser JE, Wang X and Akey CW: Three-dimensional structure of the apoptosome: Implications for assembly, procaspase-9 binding and activation. Mol Cell 9: 423-432, 2002.

4. Mei H, Sun S, Bai Y, Chen Y, Chai R and Li H: Reduced mtDNA copy number increases the sensitivity of tumor cells to chemotherapeutic drugs. Cell Death Dis 6: e1710, 2015.

5. Jornayvaz FR and Shulman GI: Regulation of mitochondrial biogenesis. Essays Biochem 47: 69-84, 2010.

6. van Tienen FH, Lindsey PJ, van der Kallen CJ and Smeets HJ: Prolonged Nrf1 overexpression triggers adipocyte inflammation and insulin resistance. J Cell Biochem 111: 1575-1585, 2010.

7. Benner C, Konovalov S, Mackintosh C, Hutt KR, Stunnenberg R and Garcia-Bassets I: Decoding a signature-based model of transcription cofactor recruitment dictated by cardinal cis-regulatory elements in proximal promoter regions. PLoS Genet 9: e1003906, 2013.

8. Lin CS, Lee HT, Lee SY, Shen YA, Wang LS, Chen YJ and Wei YH: High mitochondrial DNA copy number and bioenergetic function are associated with tumor invasion of esophageal squamous cell carcinoma cell lines. Int J Mol Sci 13: 11228-11246, 2012.

9. Guo J, Zheng L, Liu W, Wang X, Wang Z, Wang Z, French AJ, Kang D, Chen L, Thibodeau SN and Liu W: Frequent truncating mutation of TFAM induces mitochondrial DNA depletion and apoptotic resistance in microsatellite-unstable colorectal cancer. Cancer Res 71: 2978-2987, 2011. 
10. Vadrot N, Ghanem S, Braut F, Gavrilescu L, Pilard N, Mansouri A, Moreau R and Reyl-Desmars F: Mitochondrial DNA maintenance is regulated in human hepatoma cells by glycogen synthase kinase $3 \beta$ and p53 in response to tumor necrosis factor $\alpha$. PLoS One 7: e40879, 2012.

11. Mo M, Peng F, Wang L, Peng L, Lan G and Yu S: Roles of mitochondrial transcription factor A and microRNA-590-3p in the development of bladder cancer. Oncol Lett 6: 617-623, 2013.

12. Yao J, Zhou E, Wang Y, Xu F, Zhang D and Zhong D: microRNA-200a inhibits cell proliferation by targeting mitochondrial transcription factor A in breast cancer. DNA Cell Biol 33: 291-300, 2014.

13. Fuchinoue F, Hirotani Y, Nakanishi Y, Yamaguchi $H$, Nishimaki H, Noda H, Tang XY, Iizuka M, Amano S, Sugitani M, et al: Overexpression of PGC1 $\alpha$ and accumulation of p62 in apocrine carcinoma of the breast. Pathol Int 65: 19-26, 2015.

14. Gradishar WJ, Anderson BO, Blair SL, Burstein HJ, Cyr A, Elias AD, Farrar WB, Forero A, Giordano SH and Goldstein LJ: NCCN Clinical Practice Guidelines in Oncology: Breast Cancer, Version 3.2014. J Natl Compr Canc Netw 12: 542-590, 2014.

15. Hu J, Wang N and Wang YJ: XRCC3 and RAD51 expression are associated with clinical factors in breast cancer. PLoS One 8: e72104, 2013

16. Wolff AC, Hammond ME, Hicks DG, Dowsett $M$, McShane LM, Allison KH, Allred DC, Bartlett JM, Bilous M, Fitzgibbons $\mathrm{P}$, et al: Recommendations for human epidermal growth factor receptor 2 testing in breast cancer: American Society of Clinical Oncology/College of American Pathologists clinical practice guideline update. J Clin Oncol 31: 3997-4013, 2013.

17. Singletary SE, Allred C, Ashley P, Bassett LW, Berry D, Bland KI, Borgen PI, Clark G, Edge SB, Hayes DF, et al: Revision of the American Joint Committee on cancer staging system for breast cancer. J Clin Oncol 20: 3628-3636, 2002.

18. Sotgia F, Whitaker-Menezes D, Martinez-Outschoorn UE, Salem AF, Tsirigos A, Lamb R, Sneddon S, Hulit J, Howell A and Lisanti MP: Mitochondria 'fuel' breast cancer metabolism: Fifteen markers of mitochondrial biogenesis label epithelial cancer cells, but are excluded from adjacent stromal cells. Cell Cycle 11: 4390-4401, 2012.

19. Chatterjee A, Ronghe A, Singh B, Bhat NK, Chen J and Bhat HK: Natural antioxidants exhibit chemopreventive characteristics through the regulation of $\mathrm{CNC}$ b-Zip transcription factors in estrogen-induced breast carcinogenesis. J Biochem Mol Toxicol 28: 529-538, 2014.
20. Kang D, Kim SH and Hamasaki N: Mitochondrial transcription factor A (TFAM): Roles in maintenance of mtDNA and cellular functions. Mitochondrion 7: 39-44, 2007.

21. Larsson NG, Wang J, Wilhelmsson H, Oldfors A, Rustin P, Lewandoski M, Barsh GS and Clayton DA: Mitochondrial transcription factor A is necessary for mtDNA maintenance and embryogenesis in mice. Nat Genet 18: 231-236, 1998.

22. Ekstrand MI, Falkenberg M, Rantanen A, Park CB, Gaspari M, Hultenby K, Rustin P, Gustafsson CM and Larsson NG: Mitochondrial transcription factor A regulates mtDNA copy number in mammals. Hum Mol Genet 13: 935-944, 2004.

23. Pohjoismäki JL, Wanrooij S, Hyvärinen AK, Goffart S, Holt IJ, Spelbrink JN and Jacobs HT: Alterations to the expression level of mitochondrial transcription factor A, TFAM, modify the mode of mitochondrial DNA replication in cultured human cells. Nucleic Acids Res 34: 5815-5828, 2006.

24. Wang J, Silva JP, Gustafsson CM, Rustin P and Larsson NG: Increased in vivo apoptosis in cells lacking mitochondrial DNA gene expression. Proc Natl Acad Sci USA 98: 4038-4043, 2001.

25. Choi YS, Kim S and Pak YK: Mitochondrial transcription factor A (mtTFA) and diabetes. Diabetes Res Clin Pract 54 (Suppl 2): S3-S9, 2001.

26. Yoshida $\mathrm{Y}$, Izumi $\mathrm{H}$, Torigoe $\mathrm{T}$, Ishiguchi $\mathrm{H}$, Itoh $\mathrm{H}$, Kang D and Kohno K: P53 physically interacts with mitochondrial transcription factor A and differentially regulates binding to damaged DNA. Cancer Res 63: 3729-3734, 2003.

27. Piantadosi CA and Suliman HB: Mitochondrial transcription factor A induction by redox activation of nuclear respiratory factor 1. J Biol Chem 281: 324-333, 2006.

28. Choi YS, Kim S, Kyu Lee H, Lee KU and Pak YK: In vitro methylation of nuclear respiratory factor-1 binding site suppresses the promoter activity of mitochondrial transcription factor A. Biochem Biophys Res Commun 314: 118-122, 2004.

29. Kelly DP and Scarpulla RC: Transcriptional regulatory circuits controlling mitochondrial biogenesis and function. Genes Dev 18: 357-368, 2004.

This work is licensed under a Creative Commons Attribution-NonCommercial-NoDerivatives 4.0 International (CC BY-NC-ND 4.0) License. 